\title{
ORDERED FIELD VALUED CONTINUOUS FUNCTIONS WITH COUNTABLE RANGE
}

\author{
Sudip Kumar Acharyya, Atasi Deb Ray, and Pratip Nandi \\ Abstract. For a Hausdorff zero-dimensional topological space $X$ and a totally \\ ordered field $F$ with interval topology, let $C_{c}(X, F)$ be the ring of all $F$-valued \\ continuous functions on $X$ with countable range. It is proved that if $F$ is \\ either an uncountable field or countable subfield of $\mathbb{R}$, then the structure space \\ of $C_{c}(X, F)$ is $\beta_{0} X$, the Banaschewski Compactification of $X$. The ideals \\ $\left\{O_{c}^{p, F}: p \in \beta_{0} X\right\}$ in $C_{c}(X, F)$ are introduced as modified countable analogue \\ of the ideals $\left\{O^{p}: p \in \beta X\right\}$ in $C(X)$. It is realized that $C_{c}(X, F) \cap C_{K}(X, F)=$ \\ $\bigcap_{p \in \beta_{0} X \backslash X} O_{c}^{p, F}$, this may be called a countable analogue of the well-known \\ formula $C_{K}(X)=\bigcap_{p \in \beta X \backslash X} O^{p}$ in $C(X)$. Furthermore, it is shown that the \\ hypothesis $C_{C}(X, F)$ is a Von-Neumann regular ring is equivalent to amongst \\ others the condition that $X$ is a $P$-space.
}

\section{Introduction}

Let $F$ be a totally ordered field equipped with its ordered topology. For any topological space $X$, suppose $C(X, F)$ is the set of all $F$-valued continuous functions on $X$. This later set becomes a commutative lattice ordered ring with unity, if the operations are defined pointwise on $X$. As in classical scenario with $F=\mathbb{R}$, there is already discovered an interplay existing between the topological structure of $X$ and the algebraic ring and order structure of $C(X, F)$ and a few of its chosen subrings. In order to study this interaction, one can stick to a well-chosen class of spaces viz. the so-called completely $F$-regular topological spaces or in brief $C F R$ spaces. $X$ is called $C F R$ space if it is Hausdorff and points and closed sets in $X$ could be separated by $F$-valued continuous functions in an obvious manner. Problems of this kind are addressed in [1, [2], [3], 4], 5], [9. It turns out that with $F \neq \mathbb{R}, C F R$ spaces are precisely zero-dimensional spaces. Thus zero-dimensionality on $X$ can be realized as a kind of separation axiom effected by $F$-valued continuous functions on $X$. In the present article, we intend to examine the countable analogue of the ring $C(X, F)$ vis-a-vis the corresponding class of spaces $X$. Towards that end, we let

2010 Mathematics Subject Classification. Primary 54C40; Secondary 46E25.

Key words and phrases. totally ordered field, zero-dimensional space, Banaschewski Compactification, $Z_{F}^{c}$-ideal, $P$-space, $m_{F}^{c}$ tpology.

The third author thanks the CSIR, New Delhi 110001, India, for financial support. 
$C_{c}(X, F)=\{f \in C(X, F): f(X)$ is a countable subset of $F\}$. Then $C_{c}(X, F)$ is a subring as well as a sublattice of $C(X, F)$. It is interesting to note that spaces $X$ in which points and closed sets can be separated by functions in $C_{c}(X, F)$ are exactly zero-dimensional also [Theorem 2.9]. Furthermore, the set of all maximal ideals in $C_{c}(X, F)$ endowed with the well-known Hull-Kernel topology (also known as the structure space of $\left.C_{c}(X, F)\right)$ turns out to be homeomorphic to the Banaschewski Compactification $\beta_{0} X$ of $X$ [Theorem 2.18]. To achieve this result, we have to put certain restriction on the nature of the totally ordered field $F$ viz. that $F$ is either an uncountable field or a countable subfield of $\mathbb{R}$. A special case of this result choosing $F=\mathbb{R}$ reads: the structure space of the ring $C_{C}(X)$ consisting all real-valued continuous functions on $X$ with countable range is $\beta_{0} X$, which is Remark 3.6 in [7]. Since the maximal ideals of $C_{c}(X, F)$ can be indexed by virtue of the points of $\beta_{0} X$, it is not surprising that a complete description of these ideals can be given by the family $\left\{M_{c}^{p, F}: p \in \beta_{0} X\right\}$, where $M_{c}^{p, F}=\{f \in$ $\left.C_{c}(X, F): p \in \operatorname{cl}_{\beta_{0} X} Z_{c}(f)\right\}$, here $Z_{c}(f)=\{x \in X: f(x)=0\}$ stands for the zero set of $f$ [Remark 2.21]. This is analogous to the GelfandKolmogorov Theorem 7.3 [12. Also, this places Theorem 4.8 [11] on a wider setting. As a natural companion of $M_{c}^{p, F}$, we introduce the ideal $O_{c}^{p, F}=\left\{f \in M_{c}^{p, F}: c l_{\beta_{0} X} Z_{c}(f)\right.$ is a neighbourhood of $p$ in $\left.\beta_{0} X\right\}$. Amongst other facts connecting these two classes of ideals in $C_{c}(X, F)$, we have realized that the ideals that lie between $O_{c}^{p, F}$ and $M_{c}^{p, F}$ are precisely those that extend to unique maximal ideals in $C_{c}(X, F)$ [Theorem 3.1(4)]. This may be called the modified countable counterpart of Theorem 7.13 in [12. Also see Lemma 4.11 in [7] in this connection. If $C_{K}^{c}(X, F)=\left\{f \in C_{c}(X, F): c l_{X}\left(X-Z_{c}(f)\right)\right.$ is compact $\}$, then we have found out a formula for this ring in terms of the ideals $O_{c}^{p, F}$ as follows : $C_{K}^{c}(X, F)=\bigcap_{p \in \beta_{0} X \backslash X} O_{c}^{p, F}$ [in Theorem 3.5, compare with the Theorem 3.9, [4]]. This we may call the appropriate modified countable analogue of the well-known formula in $C(X)$ which says that $C_{K}(X)=\bigcap_{p \in \beta X \backslash X} O^{p}[7 E,[\mathbf{1 2}]]$. The above-mentioned results constitute technical section 2 and 3 of the present article.

In the final section 4 of this article, we have examined several possible consequences of the hypothesis that $C_{c}(X, F)$ is a Von-Neumann regular ring with $F$, either an uncountable field or a countable subfield of $\mathbb{R}$. To aid to this examination, we introduce $m_{c}^{F}$-topology on $C_{c}(X, F)$ as a modified version of $m_{c}$-topology on $C_{c}(X)$ already introduced in 6]. We establish amongst a host of necessary and sufficient conditions that $C_{c}(X, F)$ is a Von-Neumann regular ring if and only if each ideal in $C_{c}(X, F)$ is closed in the $m_{c}^{F}$-topology if and only if $X$ is a $P$-space. This places theorem 3.9 in [6] on a wider settings, and we may call it a modified countable analogue of the well-known fact that $X$ is a $P$-space when and only when each ideal in $C(X)$ is closed in the $m$-topology $[7 Q 4,[\mathbf{1 2}]$. 


\section{Duality between ideals in $C_{c}(X, F)$ and $Z_{F_{c}}$-filters on $X$}

Notation 2.1. In spite of the difference of notations, we write for $f \in C_{c}(X, F), Z_{c}(f) \equiv\{x \in X: f(x)=0\} \equiv Z(f)$

Let $Z_{c}(X, F)=\left\{Z_{c}(f): f \in C_{c}(X, F)\right\}$.

An ideal unmodified in a ring will always stand for a proper ideal.

DeFinition 2.2. A filter of zero sets in the family $Z_{c}(X, F)$ is called a $Z_{F_{c}}$-filter on $X$. A $Z_{F_{c}}$-filter on $X$ is called a $Z_{F_{c}}$-ultrafilter on $X$ if it is not properly contained in any $Z_{F_{c}}-$ filter on $X$.

REMARK 2.3. A straight forward use of Zorn's Lemma tells that a $Z_{F_{c}}$-filter on $X$ extends to a $Z_{F_{c}}$-ultrafilter on $X$. Furthermore any subfamily of $Z_{c}(X, F)$ with finite intersection property can be extended to a $Z_{F_{c}}$-ultrafilter on $X$.

The following results correlating $Z_{F_{c}}$-filters on $X$ and ideals in $C_{c}(X, F)$ can be established by using routine arguments.

THEOREM 2.4.

(1) If $I$ is an ideal in $C_{c}(X, F)$, then $Z_{F, C}[I]=\left\{Z_{c}(f): f \in I\right\}$ is a $Z_{F_{c}}$-filter on $X$. Dually for a $Z_{F_{c}}$-filter $\mathcal{F}$ on $X, Z_{F C}^{-1}[\mathcal{F}]=$ $\left\{f \in C_{c}(X, F): Z_{c}(f) \in \mathcal{F}\right\}$ is an ideal in $C_{c}(X, F)$.

(2) If $M$ is a maximal ideal in $C_{c}(X, F)$, then $Z_{F, C}[M]$ is a $Z_{F_{c}}-$ ultrafilter on $X$. If $\mathcal{U}$ is a $Z_{F_{c}}-$ ultrafilter on $X$, then $Z_{F, C}^{-1}[\mathcal{U}]$ is a maximal ideal in $C_{c}(X, F)$.

Definition 2.5. An ideal $I$ in $C_{c}(X, F)$ is called $Z_{F_{c}}$-ideal if $Z_{F, C}^{-1}\left[Z_{F, C}[I]\right]=I$

It follows from Theorem 2.4(2) that each maximal ideal in $C_{c}(X, F)$ is a $Z_{F_{c}}$-ideal. Hence the assignment $: M \rightarrow Z_{F, C}[M]$ establish a oneto-one correspondence between the maximal ideals in $C_{c}(X, F)$ and the $Z_{F_{c}}$-ultrafilters on $X$.

The following propositions can be easily established on using the arguments adopted in Chapter 2 and Chapter 4 of $[\mathbf{1 2}$ in a straight forward manner.

TheOREm 2.6. A $Z_{F_{c}}$-ideal $I$ in $C_{c}(X, F)$ is a prime ideal if and only if it contains a prime ideal. Hence each prime ideal in $C_{c}(X, F)$ extends to a unique maximal ideal, in other words, $C_{c}(X, F)$ is a Gelfand ring.

TheOREm 2.7. The complete list of fixed maximal ideals in $C_{c}(X, F)$ is given by $\left\{M_{p, F}^{c}: p \in X\right\}$ where $M_{p, F}^{c}=\left\{f \in C_{c}(X, F): f(p)=0\right\}$. An ideal $I$ in $C_{c}(X, F)$ is called fixed if $\bigcap_{f \in I} Z(f) \neq \phi$.

DeFinition 2.8. $X$ is called countably completely $F$-regular or in brief $C C F R$ space if it is Hausdorff and given a closed set $K$ in $X$ and a point $x \in X \backslash K$, there exists $f \in C_{c}(X, F)$ such that $f(x)=0$ and $f(K)=1$. 
It is clear that a $C C F R$ space is $C F R$.

A $C F R$ space with $F \neq \mathbb{R}$ is zero-dimensional by Theorem 2.3 in [4]. A $C C F R$ space with $F=\mathbb{R}$ is the same as $C$-completely regular space introduced in $1 \mathbf{1 1}$ and is hence zero-dimensional space by Proposition 4.4 in [11. Thus for all choices of the field $F$, a $C C F R$ space is zerodimensional. Conversely, it is easy to prove that a zero-dimensional space $X$ is $C C F R$ for any totally ordered field $F$. Thus, the following result comes out immediately.

THEOREM 2.9. The statements written below are equivalent for a Hausdorff space $X$ and for any totally ordered field $F$ :

(1) $X$ is zero-dimensional.

(2) $X$ is CCFR.

(3) $Z_{c}(X, F)$ is a base for closed sets in $X$.

The following result tells that as in the classical situation with $F=$ $\mathbb{R}$, in the study of the ring $C_{c}(X, F)$, one can assume without loss of generality that the ambient space $X$ is $C C F R$, i.e., zero-dimensional.

TheOREM 2.10. Let $X$ be a topological space and $F$, a totally ordered field. Then it is possible to construct a zero-dimensional Hausdorff space $Y$ such that the ring $C_{c}(X, F)$ is isomorphic to the ring $C_{c}(Y, F)$

We need the following two subsidiary results to prove this theorem.

Lemma 2.11. A Hausdorff space $X$ is zero-dimensional if and only if given any ordered field $F$, there exists a subfamily $\mathcal{S} \subset F_{c}^{X}=\{f \in$ $F^{X}: f(X)$ is countable set $\}$, which determines the topology on $X$ in the sense that, the given topology on $X$ is the smallest one with respect to which each function in $\mathcal{S}$ is continuous.

The proof of this lemma can be accomplished by closely following the arguments in Theorem 3.7 in [12] and using Theorem 2.9.

Lemma 2.12. Suppose $X$ is a topological space whose topology is determined by a subfamily $\mathcal{S}$ of $F_{c}^{X}$. Then for a topological space $Y$, a function $h: Y \rightarrow X$ is continuous if and only if for each $g \in \mathcal{S}$, $g \circ h: Y \rightarrow F$ is a continuous map.

The proof of the last lemma is analogous to that of Theorem 3.8 in [12].

Proof. of the main theorem : Define a binary relation ' $\sim$ ' on $X$ as follows : for $x, y \in X, x \sim y$ if and only if for each $f \in$ $C_{c}(X, F), f(x)=f(y)$.

Suppose $Y=\{[x]: x \in X\}$, the set of all corresponding disjoint classes. Let $\tau: X \rightarrow Y$ be the canonical map given by $\tau(x)=[x]$. Each $f \in C_{c}(X, F)$ gives rise to a function $g_{f}: Y \rightarrow F$ as follows : 
$g_{f}[x]=f(x)$.

Let $\mathcal{S}=\left\{g_{f}: f \in C_{c}(X, F)\right\}$. Then $\mathcal{S} \subset F_{c}^{Y}$. Equip $Y$ with the smallest topology, which makes each function in $\mathcal{S}$ continuous. It follows from the Lemma 2.11, that $Y$ is a zero-dimensional space and it is easy to check that $Y$ is Hausdorff. The continuity of $\tau$ follows from Lemma 2.12. Now by the following arguments in Theorem 3.9 in [12], we can prove that the assignment $: C_{c}(Y, F) \rightarrow C_{c}(X, F): g \rightarrow g \circ \tau$ is an isomorphism onto $C_{c}(X, F)$.

The following result is a countable counterpart of a portion of Theorem 4.11 in $\mathbf{1 2}$.

THEOREM 2.13. For a zero-dimensional Hausdorff space $X$ and a totally ordered field $F$, the following three statements are equivalent:

(1) $X$ is compact.

(2) Each ideal in $C_{c}(X, F)$ is fixed.

(3) Each maximal ideal in $C_{c}(X, F)$ is fixed.

Proof. (1) $\Longrightarrow(2)$ and $(2) \Longrightarrow(3)$ are trivial. We prove $(3) \Longrightarrow(1)$ : Let (3) be true.

Suppose $\mathcal{B}$ is a subfamily of $Z_{c}(X, F)$ with finite intersection property. Since $Z_{c}(X, F)$ is a base for the closed sets in $X$ (vide Theorem 2.9), it suffices to show that $\bigcap \mathcal{B} \neq \phi$.

Indeed $\mathcal{B}$ can be extended to a $Z_{F_{c}}$-ultrafilter $\mathcal{U}$ on $X$. In view of Theorem 2.4, we can write $\mathcal{U}=Z_{F, C}[M]$ for a maximal ideal $M$ in $C_{c}(X, F)$. Hence $\bigcap \mathcal{B} \supset \bigcap \mathcal{U} \neq \phi$.

Before proceeding further, we reproduce below the following basic facts about the structure space of a commutative ring with unity from $7 M,[12$.

Let $A$ be a commutative ring with unity and $\mathcal{M}(A)$, the set of all maximal ideals in $A$. For each $a \in A$, let $\mathcal{M}_{a}=\{M \in \mathcal{M}(A): a \in M\}$. Then the family $\left\{\mathcal{M}_{a}: a \in A\right\}$ constitutes a base for the closed sets of some topology $\tau$ on $\mathcal{M}(A)$. The topological space $(\mathcal{M}(A), \tau)$ is known as the structure space of $A$ and is a compact $T_{1}$ space. If $A$ is a Gelfand ring, then it is established in Theorem 1.2, [13] that $\tau$ is a Hausdorff topology on $\mathcal{M}(A)$. The closure of a subset $\mathcal{M}_{0}$ of $\mathcal{M}(A)$ is given by : $\overline{\mathcal{M}_{0}}=\left\{M \in \mathcal{M}(A): M \supset \bigcap \mathcal{M}_{0}\right\} \equiv$ the hull of the kernel of $\mathcal{M}_{0}$. [This is the reason why $\tau$ is also called the hull-kernel topology on $\mathcal{M}(A)$ ].

Let us denote the structure space of the $\operatorname{ring} C_{c}(X, F)$ by the notation $\mathcal{M}_{c}(X, F)$. Since $C_{c}(X, F)$ is a Gelfand ring, already verified in Theorem 2.6, it follows that $\mathcal{M}_{c}(X, F)$ is a compact Hausdorff space. From now on, we assume that $X$ is Hausdorff and zero-dimensional, and we will stick to this hypothesis throughout this article. It follows that the assignment $\psi: X \rightarrow \mathcal{M}_{c}(X, F)$ given by $\psi(p)=M_{p, F}^{c}$ is one-to-one. 
Furthermore for any $f \in C_{c}(X, F)$,

$$
\psi\left(Z_{c}(f)\right)=\left\{M_{p, F}^{c}: f \in M_{p, F}^{c}\right\}=\mathcal{M}_{f} \cap \psi(X)
$$

where $\mathcal{M}_{f}=\left\{M \in \mathcal{M}_{c}(X, F): f \in M\right\}$.

This shows that $\psi$ exchanges the basic closed sets of the two spaces $X$ and $\psi(X)$. Finally,

$$
\begin{aligned}
\overline{\psi(X)} & =\left\{M \in \mathcal{M}_{c}(X, F): M \supset \bigcap \psi(X)\right\} \\
& =\left\{M \in \mathcal{M}_{c}(X, F): M \supset \bigcap_{p \in X}\left\{M_{p, F}^{c}\right\}=\{0\}\right\} \\
& =\mathcal{M}_{c}(X, F)
\end{aligned}
$$

This leads to the following proposition :

Theorem 2.14. The map $\psi: X \rightarrow \mathcal{M}_{c}(X, F)$ given by $\psi(p)=$ $M_{p, F}^{c}$ defines a topological embedding of $X$ onto a dense subspace of $\mathcal{M}_{c}(X, F)$. In a more formal language, the pair $\left(\psi, \mathcal{M}_{c}(X, F)\right)$ is a Hausdorff Compactification of $X$.

The next result shows that the last-mentioned compactification enjoys a special extension property.

TheOrem 2.15. The compactification $\left(\psi, \mathcal{M}_{c}(X, F)\right)$ enjoys the $C$-extension property (see Definition 2.5 in [6] ) in the following sense, given a compact Hausdorff zero-dimensional space $Y$ and a continuous map $f: X \rightarrow Y$, there can be defined a continuous map $f^{c}$ : $\mathcal{M}_{c}(X, F) \rightarrow Y$ with the following property : $f^{c} \circ \psi=f$.

Proof. This can be accomplished by closely adapting the arguments made in the second paragraph in the proof of the Theorem 2.7 in [6]. However, to make the paper self-contained, we sketch a brief outline of the main points of its proof.

Let $M \in \mathcal{M}_{c}(X, F)$. Define as in [6], $\widetilde{M}=\left\{g \in C_{c}(Y, F): g \circ f \in M\right\}$. Then $\widetilde{M}$ is a prime ideal in $C_{c}(Y, F)$. Since $C_{c}(Y, F)$ is Gelfand ring and $Y$ is compact and zero-dimensional, it follows from Theorem 2.13 that there exists a unique $y \in Y$ such that $\bigcap_{g \in \widetilde{M}} Z_{c}(g)=\{y\}$. Set $f^{c}(M)=y$. Then $f^{c}: \mathcal{M}_{c}(X, F) \rightarrow Y$ is the desired continuous map.

REMARK 2.16. If the structure space $\mathcal{M}_{c}(X, F)$ of $C_{c}(X, F)$ is zerodimensional, then $\left(\psi, \mathcal{M}_{c}(X, F)\right)$ is topologically equivalent to the Banaschewski Compactification $\beta_{0} X$ of $X$. [see the comments after Definition 2.5 in [6]].

We shall now impose a condition on $F$; sufficient to make $\mathcal{M}_{c}(X, F)$ zero-dimensional.

TheOREM 2.17. Suppose the totally ordered field $F$ is either uncountable or a countable subfield of $\mathbb{R}$. Then given $f \in C_{c}(X, F)$, there 
exists an idempotent $e \in C_{c}(X, F)$ such that $e$ is a multiple of $f$ and $(1-e)$ is a multiple of $(1-f)$

Proof. We prove this theorem with the assumption that $F$ is uncountable. The proof for the case when $F$ is a countable subfield of $\mathbb{R}$ can be accomplished on using some analogous arguments. We first assert that the interval $[0,1]=\{\alpha \in F: 0 \leq \alpha \leq 1\}$ is an uncountable set. This is immediate if $F$ is Archimedean ordered because in that case $F^{+}=\{\alpha \in F: \alpha \geq 0\}=\bigcup_{n \in \mathbb{N} \cup\{0\}}[n, n+1]$ and for each $n \in \mathbb{N} \cup\{0\},[n, n+1]$ is equipotent with $[0,1]$ through the translation map : $\alpha \rightarrow(\alpha+n), \alpha \in[0,1]$. Now suppose that $F$ is non-Archimedean ordered field. If possible let $[0,1]$ be a countable set. Then the set $F^{+} \backslash \bigcup_{n \in \mathbb{N} \cup\{0\}}[n, n+1]$ becomes an uncountable set, which means that the set of all infinitely large members of $F$ make an uncountable set. Consequently, the set $I=\left\{\alpha \in F^{+}: 0<\alpha<\frac{1}{n}\right.$ for each $\left.n \in \mathbb{N}\right\}$ comprising of the infinitely small members of $F$ is an uncountable set. But it is easy to see that $I \subset(0,1)$ and therefore $(0,1)$ turns out to be an uncountable set - a contradiction. Thus it is proved that $[0,1]$ is an uncountable set [and consequently for any $\alpha>0$ in $F,(0, \alpha)$ becomes an uncountable set]. So we can choose $r \in(0,1)$ such that $r \notin f(X)$. Let, $W=\{x \in X: f(x)<r\}=\{x \in X: f(x) \leq r\}$ and so $X \backslash W=\{x \in X: f(x)>r\}=\{x \in X: f(x) \geq r\}$. It is clear that $W$ and $X \backslash W$ are clopen sets in $X$ and the function $e: X \rightarrow F$ defined by $e(W)=\{0\}$ and $e(X \backslash W)=\{1\}$ is an idempotent in $C_{c}(X, F)$. We see that $Z_{c}(f) \subset Z_{c}(e)$ and $Z_{c}(1-f) \subset Z_{c}(1-e)$ and we can say that $Z_{c}(e)$ is a neighbourhood of $Z_{c}(f)$ and $Z_{c}(1-e)$ is a neighbourhood of $Z_{c}(1-f)$ in the space $X$. Hence $e$ is a multiple of $f$ and $(1-e)$ is a multiple of $(1-f)$. [compare with the arguments made in Remark 3.6 in [7]].

TheOrem 2.18. The structure space $\mathcal{M}_{c}(X, F)$ of $C_{c}(X, F)$ is zerodimensional and hence $\mathcal{M}_{c}(X, F)=\beta_{0} X$.

[Here $F$ is either uncountable or a countable subfield of $\mathbb{R}$ ]

Proof. Recall the notation for $f \in C_{c}(X, F), \mathcal{M}_{f}=\left\{M \in \mathcal{M}_{c}(X\right.$, $F): f \in M\}$. Suppose $M \in \mathcal{M}_{c}(X, F)$ is such that $M \notin \mathcal{M}_{f}$. It suffices to find out an idempotent $e$ in $C_{c}(X, F)$ with the property : $\mathcal{M}_{f} \subset \mathcal{M}_{e}$ and $M \notin \mathcal{M}_{e}$. The simple reason is that $e .(1-e)=e-e^{2}=$ $e-e=0$ and hence $\mathcal{M}_{e}=\mathcal{M}_{c}(X, F) \backslash \mathcal{M}_{(1-e)}$, consequently $\mathcal{M}_{e}$ is a clopen set in $\mathcal{M}_{c}(X, F)$. Now towards finding out such an idempotent let us observe that $M \notin \mathcal{M}_{f}$ implies that $f \notin M$, which further implies that $\langle f, M\rangle=C_{c}(X, F)$. Hence we can write : $1=f . h+g$, where $h \in C_{c}(X, F)$ and $g \in M$. By Theorem 2.17, there exists an idempotent $e$ in $C_{c}(X, F)$ such that $e=g_{1} . g$ and $(1-e)=g_{2} .(1-g)$, where $g_{1}, g_{2} \in C_{c}(X, F)$. Now let $N \in \mathcal{M}_{f}$, then $f \in N$ and so $f . h \in N$, which implies that $(1-g) \in N$ consequently $(1-e) \in N$. Therefore 
$e \notin N$, which means that $N \notin \mathcal{M}_{e}$, i.e., $N \in \mathcal{M}_{c}(X, F) \backslash \mathcal{M}_{e}$. Again since $g \in M$, it follows that $e \in M$, thus $M \in \mathcal{M}_{e}$.

REmark 2.19. On choosing $F=\mathbb{R}$ and $X=\mathbb{Q}$ in the above Theorem 2.18 , we get that $\beta_{0} \mathbb{Q}=$ structure space of $C(\mathbb{Q}, \mathbb{R})=\beta \mathbb{Q}$. Thus $\beta \mathbb{Q}$ becomes zero-dimensional, i.e., $\mathbb{Q}$ is strongly zero-dimensional. This is a standard result in General Topology - indeed a Lindelöf zerodimensional space is strongly zero-dimensional. [Theorem 6.2.7, [10]].

One of the major achievements in the theory of $C(X)$ is that a complete description of the maximal ideals in this ring can be given. This is a remark made in the beginning of Chapter 6 in [12. In order to give such a description, it becomes convenient to archive $\beta X$ as the space of $Z$-ultrafilter on $X$ equipped with the Stone- topology and formal construction of such a thing is dealt in rigorously in Chapter 6 in 12. We follow the same technique in order to furnish an explicit description of maximal ideals in $C_{c}(X, F)$.

For each $p \in X$, let $A_{p, F}^{c}=\left\{Z \in Z_{c}(X, F): p \in Z\right\} \equiv Z_{F, C}\left[M_{p, F}^{c}\right]$. Thus $X$ is a readymade index set for the family of fixed $Z_{F_{c}}-$ ultrafilters on $X$. As in Chapter 6, [12, we extend the set $X$ to a set $\alpha X$ to serve as an index set for the family of all $Z_{F_{c}}$-ultrafilters on $X$. For $p \in \alpha X$, let the corresponding $Z_{F_{c}}$-ultrafilter be designated as $A_{c}^{p, F}$ with the understanding that if $p \in X$, then $A_{c}^{p, F}=A_{p, F}^{c}$.

For $Z \in Z_{c}(X, F)$, let $\bar{Z}=\left\{p \in \alpha X: Z \in A_{c}^{p, F}\right\}$. Then $\{\bar{Z}: z \in$ $\left.Z_{c}(X, F)\right\}$ makes a base for the closed sets of some topology on $\alpha X$ in which for $Z \in Z_{c}(X, F), \bar{Z}=c l_{\alpha X} Z$. Furthermore, for $Z_{1}, Z_{2} \in$ $Z_{c}(X, F), \overline{Z_{1} \cap Z_{2}}=\overline{Z_{1}} \cap \overline{Z_{2}}$ and $\alpha X$ becomes a compact Hausdorff space containing $X$ as a dense subset. Also given a point $p \in \alpha X, A_{c}^{p, F}$ is the unique $Z_{F_{c}}$-ultrafilter on $X$ which converges to $p$ and finally $\alpha X$ possesses the $C$-extension property meaning that if $Y$ is a compact Hausdorff zero-dimensional space and $f: X \rightarrow Y$, a continuous map, then $f$ can be extended to a continuous map $f^{\#}: \alpha X \rightarrow Y$. All these facts can be realized just by closely following the arguments in Chapter 6 in [12].

TheOREM 2.20. The space $\alpha X$ is a zero-dimensional space.

[Blanket assumption: $F$ is either an uncountable field or a countable subfield of $\mathbb{R}$ ]

Proof. Let $p \in \alpha X$ and $Z \in Z_{c}(X, F)$ be such that $p \notin \bar{Z}$. It suffices to find out a clopen set $K$ in $\alpha X$ such that $\bar{Z} \subset K$ and $p \notin K$.

Now $p \notin \bar{Z} \Longrightarrow Z \notin A_{c}^{p, F}$. Since $A_{c}^{p, F}$ is a $Z_{F_{c}}$-ultrafilter, this implies that there exists $Z^{*} \in A_{c}^{p, F}$ such that $Z \cap Z^{*}=\phi$. Hence there exists $f \in C_{c}(X, F)$ such that $f: X \rightarrow[0,1]$ in $F$ such that $f\left(Z^{*}\right)=\{0\}$ and $f(Z)=\{1\}$. Using the hypothesis that $F$ is an uncountable field, and take note of the arguments in the proof of the Theorem 2.17, we can 
find out an $r \in(0,1)$ in $F$ such that $r \notin f(X)$ [analogous arguments can be made if $F$ is a countable subfield of $\mathbb{R}$.

Let $K=\{x \in X: f(x)>r\}=\{x \in X: f(x) \geq r\}$. Then $K$ is a clopen set in $X$ containing $Z$ and therefore $\bar{Z} \subset K$. Now $Z^{*} \subset X \backslash K$ implies $Z^{*} \cap K=\phi$ and hence $\overline{Z^{*}} \cap \bar{K}=\phi$, i.e., $\overline{Z^{*}} \cap K=\phi$. Since $Z^{*} \in A_{c}^{p, F}$ and therefore $p \in Z^{*}$, this further implies that $p \notin K$.

REMARK 2.21. Since $\alpha X$ enjoys the $C$-extension property and is zero-dimensional, it follows from Definition 2.5 in [6] that $\alpha X$ is essentially the same as $\beta_{0} X$, the Banaschewski Compactification of $X$ and hence we can write for any $p \in \beta_{0} X$ and $Z \in Z_{c}(X, F), Z \in A_{c}^{p, F}$ if and only if $p \in c l_{\beta_{0} X}$. If we now write $M_{c}^{p, F}=Z_{F, C}^{-1}\left[A_{c}^{p, F}\right]$, then this becomes a maximal ideal in $C_{c}(X, F)$. Since by Theorem $2.4(2)$, there is already realized a one-to-one correspondence between maximal ideals in $C_{c}(X, F)$ and $Z_{F_{c}}$-ultrafilters on $X$ via the map $M \rightarrow Z_{F, C}[M]$, a complete description of the maximal ideals in $C_{c}(X, F)$ is given by the list $\left\{M_{c}^{p, F}: p \in \beta_{0} X\right\}$ where $M_{c}^{p, F}=\left\{f \in c_{c}(X, F): p \in c l_{\beta_{0} X} Z_{c}(f)\right\}$

\section{The ideals $O_{c}^{p, F}$ and a formula for $C_{K}^{c}(X, F)$}

For each $p \in \beta_{0} X$, set

$O_{c}^{p, F}=\left\{f \in C_{c}(X, F): c_{\beta_{0} X} Z_{c}(f)\right.$ is a neighbourhood of $p$ in $\left.\beta_{0} X\right\}$

Then the following facts come out as modified countable analogue of the relations between the ideals $M^{p}$ and $O^{p}$ in the classical scenario recorded in 7.12, 7.13, 7.15 in [12]. Also see Lemma 4.11 in [7] in this connection.

TheOREM 3.1. Let the ordered field $F$ be either uncountable or a countable subfield of $\mathbb{R}$. Then for a zero-dimensional Hausdorff space $X$, the following statements are true:

(1) $O_{c}^{p, F}$ is a $Z_{F_{c}}$-ideal in $C_{c}(X, F)$ contained in $M_{c}^{p, F}$.

(2) $O_{c}^{p, F}=\left\{f \in C_{c}(X, F)\right.$ : there exists an open neighbourhood $V$ of $p$ in $\beta_{0} X$ such that $\left.Z_{c}(f) \supset V \cap X\right\}$.

(3) For $p \in \beta_{0} X$ and $f \in C_{c}(X, F), f \in O_{c}^{p, F}$ if and only if there exists $g \in C_{c}(X, F) \backslash M_{c}^{p, F}$ such that $f . g=0$, hence each nonzero element in $O_{c}^{p, F}$ is a divisor of zero in $C_{c}(X, F)$. Indeed $O_{c}^{p, F}$ is a $z^{o}$-ideal in $C_{c}(X, F)$.

(4) An ideal $I$ in $C_{c}(X, F)$ is extendable to a unique maximal ideal if and only if there exists $p \in \beta_{0} X$ such that $O_{c}^{p, F} \subset I$.

(5) For $p \in \beta_{0} X, O_{c}^{p, F}$ is a fixed ideal if and only if $p \in X$.

Proof. The statements (1), (2) and (4) can be proved by making arguments parallel to those adopted to prove the corresponding results in the classical situation with $F=\mathbb{R}$ in Sections 7.12, 7.13, 7.15 in [12]. We prove only the statements (3) and (5).

To prove (3), let $f \in O_{c}^{p, F}$. Then by (2), there exists an open neighbourhood $V$ of $p$ in $\beta_{0} X$ such that $Z_{c}(f) \supset V \cap X$. Since $\beta_{0} X$ is zero 
dimensional, there exists a clopen set $K$ in $\beta_{0} X$ such that $\beta_{0} X \backslash V \subset K$ and $p \notin K$. The function $h: \beta_{0} X \rightarrow F$, defined by $h(K)=\{0\}$ and $h\left(\beta_{0} X \backslash K\right)=\{1\}$ belongs to $C_{c}\left(\beta_{0} X, F\right)$. Take $g=\left.h\right|_{X}$. Then $g \in C_{c}(X, F), f . g=0$ and $p \notin c l_{\beta_{0} X} Z_{c}(g)$, hence $g \notin M_{c}^{p, F}$.

Conversely let there exist $g \in C_{c}(X, F) \backslash M_{c}^{p, F}$ such that $f . g=0$. Then $p \notin c l_{\beta_{0} X} Z_{c}(g)$. Therefore there exists an open neighbourhood $V$ of $p$ in $\beta_{0} X$ such that $V \cap Z_{c}(g)=\phi$. Since $Z_{c}(f) \cup Z_{c}(g)=X$, it follows that $X \cap V \subset Z_{c}(f)$. Hence from (2), we get that $f \in O_{c}^{p, F}$.

To prove the last part of (3), we recall that an ideal $I$ in a commutative $\operatorname{ring} A$ with unity is called a $z^{o}$-ideal if for each $a \in I, P_{a} \subset I$, where $P_{a}$ is the intersection of all minimal prime ideals in $A$ containing $a$. We reproduce the following useful formula from Proposition 1.5 in [8], which is also recorded in Theorem 3.10 in [6] : if $A$ is a reduced ring meaning that 0 is the only nilpotent member of $A$, then $P_{a}=\{b \in A: \operatorname{Ann}(a) \subset \operatorname{Ann}(b)\}$, where $\operatorname{Ann}(a)=\{c \in A: a . c=0\}$ is the annihilator of $a$ in $A$. Hence for any $f \in C_{c}(X, F), P_{f} \equiv$ the intersection of all minimal prime ideals in $C_{c}(X, F)$ which contain $f=\left\{g \in C_{c}(X, F): \operatorname{Ann}(f) \subset \operatorname{Ann}(g)\right\}$.

Now to show that $O_{c}^{p, F}$ is a $z^{o}$-ideal in $C_{c}(X, F)$, for any $p \in \beta_{0} X$, choose $f \in O_{c}^{p, F}$ and $g \in P_{f}$. Therefore $A n n(f) \subset A n n(g)$. But from the result (3), we see that there exists $h \in C_{c}(X, F) \backslash M_{c}^{p, F}$ such that $f . h=0$ and hence $h \in A n n(f)$. Consequently, $h \in A n n(g)$, i.e., $g . h=0$. Thus $P_{f} \subset O_{c}^{p, F}$ and hence $O_{c}^{p, F}$ is a $z^{o}$-ideal in $C_{c}(X, F)$.

Proof of (5) : If $p \in X$, then $M_{c}^{p, F}=M_{p, F}^{c}$, a fixed ideal, hence $O_{c}^{p, F}$ is also fixed.

Now let $p \in \beta_{0} X \backslash X$. Choose $x \in X$ and a closed neighbourhood $W$ of $p$ in $\beta_{0} X$ such that $x \notin W$. Since $\beta_{0} X$ is zero-dimensional, there exists a clopen set $K$ in $\beta_{0} X$ such that $W \subset K$ and $x \notin K$. Let $g: \beta_{0} X \rightarrow F$ be defined by $g(K)=\{0\}$ and $g\left(\beta_{0} X \backslash K\right)=\{1\}$. Then $g \in C_{c}\left(\beta_{0} X, F\right)$ and hence $h=\left.g\right|_{X} \in C_{c}(X, F)$. We observe that $h(x)=1$ and $Z_{c}(h) \supset K \cap X$. It follows from the result (2) that $h \in O_{c}^{p, F}$. This proves that $O_{c}^{p, F}$ is a free ideal in $C_{c}(X, F)$

The following properties of $C_{K}^{c}(X, F)=\left\{f \in C_{c}(X, F): f\right.$ has compact support i.e. $c l_{X}\left(X \backslash Z_{c}(f)\right)$ is compact $\}$ can be established as parallel to the analogous properties of the ring $C_{K}(X)=\{f \in C(X)$ : $f$ has compact support $\}$ given in $4 D,[\mathbf{1 2}$.

Theorem 3.2. Let $X$ be Hausdorff and zero-dimensional. Then :

(1) $C_{K}^{c}(X, F) \subset C_{c}(X, F) \cap C^{*}(X, F)$, where $C^{*}(X, F)=\{f \in$ $C(X, F): c_{F} f(X)$ is compact $\}$ and equality holds if and only if $X$ is compact.

(2) If $X$ is non-compact, then $C_{K}^{c}(X, F)$ is an ideal(proper) of $C_{c}(X, F)$. 
(3) $C_{K}^{c}(X, F)$ is contained in every free ideal of $C_{c}(X, F) . C_{K}^{c}(X, F)$ itself is a free ideal of $C_{c}(X, F)$ if and only if $X$ is non-compact and locally compact.

(4) $X$ is nowhere locally compact if and only if $C_{K}^{c}(X, F)=\{0\}$ and this is the case when and only when $\beta_{0} X \backslash X$ is dense in $\beta_{0} X$. [Compare with 7F4, [12]]

REMARK 3.3. $C_{K}^{c}(X, F) \subset \bigcap\left\{O_{c}^{p, F}: p \in \beta_{0} X \backslash X\right\}$. This follows from Theorem 3.1(5) and Theorem 3.2(3).

To show that equality holds in the last inclusion relation, we need the following subsidiary result.

TheOrem 3.4. Let $f \in C_{c}(X, F)$ be such that ${ }_{\beta_{0} X} Z_{c}(f)$ is a neighbourhood of $\beta_{0} X \backslash X$. Then $f \in C_{K}^{c}(X, F)$.

Proof. It suffices to show that $\operatorname{supp}(f) \equiv l_{X}\left(X \backslash Z_{c}(f)\right)$ is closed in $\beta_{0} X$ and hence compact. As $Z_{c}(f)$ is closed in $X$, it follows that $c l_{\beta_{0} X} Z_{c}(f) \cap\left(X \backslash Z_{c}(f)\right)=\phi$. The hypothesis tells that there exists an open set $W$ in $\beta_{0} X$ such that $\beta_{0} X \backslash X \subset W \subset c l_{\beta_{0} X} Z_{c}(f)$. Hence $W \cap\left(X \backslash Z_{c}(f)\right)=\phi$, which further implies because $W$ is open in $\beta_{0} X$ that $W \cap c l_{\beta_{0} X}\left(X \backslash Z_{c}(f)\right)=\phi$. Consequently $W \cap c l_{X}\left(X \backslash Z_{c}(f)\right)=\phi$. Since $\beta_{0} X \backslash X \subset W$, it follows therefore that no point of $\beta_{0} X \backslash X$ is a limit point of $c l_{X}\left(X \backslash Z_{c}(f)\right)$ in the space $\beta_{0} X$. Thus there does not exist any limiting point of $c l_{X}\left(X \backslash Z_{c}(f)\right)$ outside it in the entire space $\beta_{0} X$. Hence $c l_{X}\left(X \backslash Z_{c}(f)\right)$ is closed in $\beta_{0} X$.

THEOREM 3.5. Let $X$ be zero-dimension and Hausdorff. Then $C_{K}^{c}(X, F)=\bigcap\left\{O_{c}^{p, F}: p \in \beta_{0} X \backslash X\right\}$

Proof. Let $f \in O_{c}^{p, F}$ for each $p \in \beta_{0} X \backslash X$. Then $c l_{\beta_{0} X} Z_{c}(f)$ is a neighbourhood of each point of $\beta_{0} X \backslash X$ in the space $\beta_{0} X$. It follows from Theorem 3.4 that $f \in C_{K}^{c}(X, F)$. Thus $\bigcap\left\{O_{c}^{p, F}: p \in\right.$ $\left.\beta_{0} X \backslash X\right\} \subset C_{K}^{c}(X, F)$. The reversed implication relation is already realized in Remark 3.3. Hence $C_{K}^{c}(X, F)=\bigcap\left\{O_{c}^{p, F}: p \in \beta_{0} X \backslash X\right\}$.

\section{Von Neumann regularity of $C_{c}(X, F)$ versus $P$-space $X$}

We recall from [3] that $X$ is called $P_{F}$-space if $C(X, F)$ is a VonNeumann regular ring. By borrowing the terminology from [1], we call a zero-dimensional space $X$, a countably $P_{F}$-space or $C P_{F}$-space if $C_{c}(X, F)$ is Von-Neumann regular ring. Thus in this terminology, $C P_{\mathbb{R}}$-spaces are precisely $C P$-spaces introduced in [11], Definition 5.1. It is still undecided whether there exist an ordered field $F$ and a zero-dimensional space $X$ for which $X$ is a $P_{F}$-space without being a $P$-space (see the comments preceding Definition 3.3 in [3] ). However, we shall prove that subject to the restrictions imposed on the field $F$, already used several times in this paper, $C P_{F}$-spaces and $P$-spaces are one and the same. We want to mention in this context that the 
zero set of a function $f$ in $C(X, F)$ may not be a $G_{\delta}$-set [see Theorem 2.2 in [3]]. In contrast, we shall show that the zero set of a function lying in $C_{c}(X, F)$ is necessarily a $G_{\delta}$-set. Before proceeding further, we make the assumption throughout the rest of this article that the ordered field $F$ is either uncountable or a countable subfield of $\mathbb{R}$.

TheOrem 4.1. A zero set $Z \in Z_{c}(X, F)$ is a $G_{\delta}$-set.

Proof. We can write $Z=Z_{c}(f)$ for some $f \geq 0$ in $C_{c}(X, F)$. Since $f(X)$ is a countable subset of $F$, we can write, $f(X) \backslash\{0\}=$ $\left\{r_{1}, r_{2}, \ldots, r_{n}, \ldots\right\} ;$ a countable set in $F^{+}$. It follows that $Z_{c}(f)=$ $\bigcap_{n=1}^{\infty} f^{-1}\left(-r_{n}, r_{n}\right)=$ a $G_{\delta}$-set in $X$.

The following results are generalized versions of Proposition 4.3, Theorem 5.5 and Corollary 5.7 in [11.

Theorem 4.2. If $A$ and $B$ are disjoint closed sets in $X$ with $A$, compact, then there exists $f \in C_{c}(X, F)$ such that $f(A)=\{0\}$ and $f(B)=\{1\}$

TheOREM 4.3. For $f \in C_{c}(X, F), Z_{c}(f)$ is a countable intersection of clopen sets in $X$

Proof. As in the proof of Theorem 4.1, we can assume $f \geq 0$ and $f(X) \backslash\{0\}=\left\{r_{1}, r_{2}, \ldots, r_{n}, \ldots\right\}$. Now if $F$ is an uncountable ordered field, then for each $n \in \mathbb{N}$, we can choose $s_{n} \in F$ such that $0<s_{n}<r_{n}$ and $s_{n} \notin\left\{r_{1}, r_{2}, \ldots, r_{n}, \ldots\right\}$. On the other hand, if $F$ is a countable subfield of $\mathbb{R}$, then we can pick up for each $n \in \mathbb{N}$ an irrational point denoted by the same symbol $s_{n}$ with the above-mentioned condition, i.e., $0<s_{n}<r_{n}$ and $s_{n} \notin\left\{r_{1}, r_{2}, \ldots, r_{n}, \ldots\right\}$. It follows that $Z_{c}(f)=$ $\bigcap_{n=1}^{\infty} f^{-1}\left(-s_{n}, s_{n}\right)=\bigcap_{n=1}^{\infty} f^{-1}\left[-s_{n}, s_{n}\right]=$ a countable intersection of clopen sets in $X$.

TheOrem 4.4. A countable intersection of clopen sets in $X$ is a zero set in $Z_{c}(X, F)$ (equivalently, a countable union of clopen sets in $X$ is a co-zero set, i.e., the complement in $X$ of a zero set in $Z_{c}(X, F)$ ).

Proof. Since in any topological space, a countable union of clopen sets can be expressed as a countable union of pairwise disjoint clopen sets, we can start with a countable family $\left\{L_{i}\right\}_{i=1}^{\infty}$ of pairwise disjoint clopen sets in $X$. For each $n \in \mathbb{N}$, define a function $e_{n}: X \rightarrow F$ as follows : $e_{n}\left(L_{n}\right)=\{1\}$ and $e_{n}\left(X \backslash L_{n}\right)=\{0\}$. Then $e_{n} \in C_{c}(X, F)$ and is an idempotent in this ring. Furthermore, it is easy to see that if $m \neq n$, then $e_{m} \cdot e_{n}=0$. Let $h(x)=\sum_{n=1}^{\infty} \frac{e_{n}(x)}{3^{n}}, x \in X$. Then $h: X \rightarrow F$ is a continuous function and $h(X) \subset\left\{0, \frac{1}{3}, \frac{1}{3^{2}}, \ldots\right\}$. Thus $h \in C_{c}(X, F)$. It is clear that $\bigcup_{n=1}^{\infty} L_{n}=X \backslash Z_{c}(h)$.

TheOREM 4.5. $Z_{c}(X, F)$ is closed under countable intersection.

Proof. Follows from Theorem 4.3 and Theorem 4.4. 
TheOREM 4.6. Suppose a compact set $K$ in $X$ is contained in a $G_{\delta}-$ set $G$. Then there exists a zero set $Z$ in $Z_{c}(X, F)$ such that $K \subset$ $Z \subset G$.

Proof. We can write $G=\bigcap_{n=1}^{\infty} W_{n}$ where each $W_{n}$ is open in $X$. For each $n \in \mathbb{N}, K$ and $X \backslash W_{n}$ are disjoint closed sets in $X$ with $K$ compact. Hence by Theorem 4.2, there exists $g_{n} \in C_{c}(X, F)$ such that $g_{n}(K)=\{0\}$ and $g_{n}\left(X \backslash W_{n}\right)=\{1\}$. It follows that $K \subset Z_{c}\left(g_{n}\right) \subset W_{n}$ for each $n \in \mathbb{N}$. Consequently, $K \subset \bigcap_{n=1}^{\infty} Z_{c}\left(g_{n}\right) \subset G$. But by Theorem 4.5, we can write $\bigcap_{n=1}^{\infty} Z_{c}\left(g_{n}\right)=Z_{c}(g)$ for some $g \in C_{c}(X, F)$. Hence $K \subset Z_{c}(g) \subset G$.

Before giving several equivalent descriptions of the defining property of $C P_{F}-$ space in the manner $4 J$ of [12] and the theorem 5.8 in [11. We like to introduce a suitable modified countable version of $m$-topology on $C(X)$ as dealt with in $2 N, \mathbf{1 2}$.

For each $g \in C_{c}(X, F)$ and a positive unit $u$ in this ring, set $M_{F}(g, u)=$ $\left\{f \in C_{c}(X, F):|f(x)-g(x)|<u(x)\right.$ for each $\left.x \in X\right\}$. Then it can be proved by routine computation that $\left\{M_{F}(g, u): g \in C_{c}(X, F), u, a\right.$ positive unit in $\left.C_{c}(X, F)\right\}$ is an open set for some topology on $C_{c}(X, F)$, which we call $m_{c}^{F}$-topology on $C_{c}(X, F)$. A special case of this topology with $F=\mathbb{R}$ is already considered in [6], Section 3. The following two can be established by making straight forward modifications in the arguments adopted to prove Theorem 3.1 and Theorem 3.7 in [6].

Theorem 4.7. Each maximal ideal in $C_{c}(X, F)$ is closed in the $m_{c}^{F}$-topology.

TheOREM 4.8. For any ideal $I$ in $C_{c}(X, F)$, its closure in $m_{c}^{F-}$ topology is given by $: \bar{I}=\bigcap\left\{M_{c}^{p, F}: p \in \beta_{0} X\right.$ and $\left.M_{c}^{p, F} \supset I\right\} \equiv$ the intersection of all the maximal ideals in $C_{c}(X, F)$ which contains $I$.

[compare with 7Q2, [12]]

THEOREM 4.9. An ideal I in $C_{c}(X, F)$ is closed in $m_{c}^{F}$-topology if and only if it is the intersection of all the maximal ideals in this ring which contains $I$.

[This follows immediately from Theorem 4.8]

We are now ready to offer a bunch of statements, each equivalent to the requirement that $X$ is a $C P_{F}-$ space.

THEOREM 4.10. Let $X$ be a zero-dimensional Hausdorff space and $F$, a totally ordered field with the property mentioned in the beginning of this section. Then the following statements are equivalent:

(1) $X$ is a $C P_{F}$-space.

(2) Each zero set in $Z_{c}(X, F)$ is open.

(3) Each ideal in $C_{c}(X, F)$ is a $Z_{F_{c}}$-ideal.

(4) For all $f, g$ in $C_{c}(X, F),<f, g>=<f^{2}+g^{2}>$.

(5) Each prime ideal in $C_{c}(X, F)$ is maximal. 
(6) For each $p \in X, M_{p, F}^{c}=O_{p, F}^{c}$.

(7) For each $p \in \beta_{0} X, M_{c}^{p, F}=O_{c}^{p, F}$.

(8) Each ideal in $C_{c}(X, F)$ is the intersection of all the maximal ideals containing it.

(9) Each $G_{\delta}$-set in $X$ is open (which eventually tells that $X$ is a P-space)

(10) Every ideal in $C_{c}(X, F)$ is closed in the $m_{c}^{F}$-topology.

Proof. Equivalence of the first eight statements can be proved by making an almost repetition of the arguments to prove the equivalence of the analogous statements in $4 J$, [12] [Also see the Theorem 5.8 in [11]]. We prove the equivalence of the statements $(2),(9),(10)$.

$(9) \Longrightarrow(2)$ is immediate because of Theorem 4.1 .

$(2) \Longrightarrow(9)$ : Let $(2)$ be true and $G$ be a non-empty $G_{\delta}$-set in $X$. Then by Theorem 4.6, for each point $x \in G$, there exists a zero set $Z_{x} \in Z_{c}(X, F)$ such that $x \in Z_{x} \subset G$. Since $Z_{x}$ is open in $X$ by (2), it follows that $x$ is an interior point of $G$. In other words, $G$ is open in $X$.

Equivalence of (8) and (10) follows from Theorem 4.9.

REMARK 4.11. On choosing $F=\mathbb{Q}$ in Theorem 4.10, we get that a zero dimensional space $X$ is a $P$-space if and only if $C(X, \mathbb{Q})$ is a Von Neumann regular ring, i.e., $X$ is a $P_{\mathbb{Q}}$-space. Thus each $P_{\mathbb{Q}}$-space is a $P$-space. But we note that, though the cofinality character of $\mathbb{Q}$ is $\omega_{0}$, it is not Cauchy complete. This improves the conclusion of the Theorem 3.5 in 3, which says that if $F$ is a Cauchy complete totally ordered field with cofinality character $\omega_{0}$, then every $P_{F}$-space is a $P$-space.

Open question : If $p \in \beta_{0} X$, then does the set of prime ideals in $C_{c}(X, F)$ that lie between $O_{c}^{p, F}$ and $M_{c}^{p, F}$ make a chain?

\section{References}

[1] S.K. Acharyya, K.C. Chattopadhyaya and P.P. Ghosh : Constructing Banaschewski Compactification Without Dedekind Completeness Axiom, Int. J. of Mathematics and Mathematical Sciences, 69 (2004), 3799-3816.

[2] S.K. Acharyya, K.C. Chattopadhyaya and P.P. Ghosh : Continuous functions with compact support, Appl. Gen. Top., 5(1) (2004), 103-113.

[3] S.K. Acharyya, K.C. Chattopadhyaya and P.P. Ghosh : A Generic method to construct Pspaces through ordered field, Southeast Asian Bulletin of Mathematics, 28 (2004), 783-790.

[4] S.K. Acharyya and P. Rooj : Structure spaces of intermediate rings of ordered field valued continuous functions, Topology Proceedings, Vol 47 (2016), 163-176.

[5] S.K. Acharyya, K.C. Chattopadhyaya and P. Rooj : A Generalized version of the rings $C_{K}(X)$ and $C_{\infty}(X)$ - an enquery about when they become Noetheri, Appl. Gen. Topology, 16(1) (2015), 81-87.

[6] S.K. Acharyya, R. Bharati and A. Deb Ray : Rings and subrings of Continuous functions with Countable range, Quaestiones Mathematicae, http://doi.org/10.2989/16073606.2020.1752322

[7] F. Azarpanah, O.A.S. Karamzadeh, Z. Keshtkar and A.R. Olfati : On Maximal Ideals of $C_{c}(X)$ and the uniformity of its localizations. Rocky Mountain J. Math., Vol. 4 No. 2, (2018), $345-384$. 
[8] F. Azarpanah, O.A.S. Karamzadeh and R.A. Aliabad : On ideals consisting entirely of zerodivisors. Comm. Algebra, 28 (2000), 1061-1073.

[9] G. Bachman, E. Beckenstein, L. Narici and S. Warner : Rings of continuous functions with values in a topological field. Trans. Amer. Math. Soc., 204 (1975), 91-112.

[10] R. Engelking : General Topology. Heldermann Verlag, Berlin, 1989.

[11] M. Ghadermazi, O.A.S. Karamzadeh and M. Namdari : On the Functionally Countable Subalgebra of $C(X)$. Rend. Sem. Mat. Univ. Padova, 129 (2013), 47-69.

[12] L. Gillman and M. Jerison : Rings of Continuous Functions. New York, Van Nostrand Reinhold Co., 1960.

[13] G.D. Marco and A. Orsatti : Commutative Rings in which every Prime Ideal is contained in a Unique Maximal Ideal. Proc. Amer. Math. Soc., 30(3) (1971), 459-466.

Department of Pure Mathematics, University of Calcutta, 35, Ballygunge Circular Road, Kolkata 700019, West Bengal, India

E-mail address: sdpacharyya@gmail.com

Department of Pure Mathematics, University of Calcutta, 35, Ballygunge CircuLAR Road, Kolkata 700019, West Bengal, India

E-mail address: debrayatasi@gmail.com

Department of Pure Mathematics, University of Calcutta, 35, Ballygunge CircuLAR Road, Kolkata 700019, West Bengal, India

E-mail address: pratipnandi10@gmail.com 